\title{
Application of evolutionary computation in automotive powertrain mount tuning
}

\author{
Anab Akanda* and Chandu Adulla \\ DaimlerChrysler Corporation Detroit, MI, USA
}

Received 3 December 2004

Revised 19 September 2005

\begin{abstract}
Engine mount tuning is a multi-disciplinary exercise since it affects Idle-shake, Road-shake and power-train noise response. Engine inertia is often used as a tuned absorber for controlling suspension resonance related road-shake issues. Last but not least, vehicle ride and handling may also be affected by mount tuning. In this work, Torque-Roll-Axis (TRA) decoupling of the rigid powertrain was used as a starting point for mount tuning. Nodal point of flexible powertrain bending was used to define the envelop for transmission mount locations. The frequency corresponding to the decoupled roll mode of the rigid powertrain was then adjusted for idle-shake and road-shake response management.

A TRA decoupling procedure, cast as a multi-objective optimization problem, was applied to a body-on-frame sport-utility vehicle powertrain system. In addition to a standard gradient based optimization algorithm, available in commercial finite element software, an evolutionary computation paradigm known as Evolutionary Strategies (ES) was used to solve the optimization problem. The primary advantages of evolutionary computation over gradient based algorithms are as follows: i) They are less likely to get trapped in local minima and less dependent on initial values of the design parameters and therefore able to handle multi-modal optimization problems unlike gradient based algorithms, ii) They produce a population of viable solutions, unlike gradient based algorithms which yields a single solution. The second advantage is very attractive in a production environment since packaging and other multi-disciplinary constraints often require multiple quality solutions for the same problem. The process outlined in this work was verified by exercising a full-vehicle finite element model. The process produced a set of production feasible powertrain mount parameters for acceptable idle and road shake performance.
\end{abstract}

\section{Introduction}

A four-wheel-drive (4WD) automotive powertrain usually consists of three major components: i) Engine, ii) Transmission and iii) Transfer case. Powertrain mount design affects multiple performance attributes of a modern automobile. Starting from vehicle ride and handling quality, low-frequency vibration, higher frequency noise to crashworthiness are all affected in some fashion by the powertrain mounting strategy. The focus of this work, however, is limited to the aspects of mounting strategy that affect low frequency vibration response due to pulsating engine idle torque and road profile induced input. Engine mounting strategies discussed here are particularly relevant to rear-wheel (or 4 wheel-drive) drive vehicles with "north-south" powertrain orientation.

As far as noise and vibration is concerned, the primary function of powertrain mounts is to isolate the vehicle body structure from any powertrain induced vibration source. Under wide/part-open-throttle conditions, modes of the flexible powertrain play a major roll in powertrain induced vehicle interior acoustic response. In this situation, isolation provided by the mounts is critical [1]. Locating the transmission mounts near or at the nodal point of the fundamental bending mode of the powertrain can significantly reduce the amount of forces transmitted to the body. Therefore, in any engine mount tuning exercise, location of the transmission mount is subjected to the above constraint.

\footnotetext{
*Corresponding author. E-mail: aa31@ daimlerchrysler.com.
} 
Under engine idle conditions, the pulsating torque generated by a multi-cylinder engine is one of the major sources of vibration. The torque frequency spectrum exhibits concentration of energy at various frequencies dictated by the engine idle rpm, number of cylinders and associated engine orders. For instance, a V6 engine would typically exhibit peaks in the frequency response related to some of the following orders: $1^{\text {st }}, 1.5,2^{\text {nd }}, 2.5$ and $3^{\text {rd }}$. In short of addressing the source of these orders caused by cylinder-to-cylinder gas pressure variation, unbalanced inertia forces etc., one is left with only two major design enablers for achieving good idle vibration response of the vehicle: i) provide maximum vibration isolation by choosing low mount rubber stiffness and ii) decouple the rigid-body modes of the powertrain. The first strategy is a fundamental principle in vibration control, while the second one is based on the following observation. In [2], Jeong et al., states: "If a system is completely decoupled in physical modes, excitation along one physical co-ordinate should excite only one mode ... Even when two or three different excitations are simultaneously applied to the powertrain, projected excitations along the physical co-ordinates may have only a few non-trivial values." In the same article, the authors provided analytical comparison of all existing decoupling schemes namely: i) Focalization method, ii) Partial elastic axis decoupling method and iii) Torque-RollAxis (TRA) decoupling method. They have proposed a new axiom for the TRA decoupling and clearly demonstrated that only the TRA mode decoupling scheme provides a complete decoupling of physical modes. They derived six equations representing the conditions for a complete decoupling of the TRA mode. If these equations are satisfied then the response of the powertrain center-of-gravity (CG) due to oscillatory torque applied about the crank-shaft will exhibit only one peak at the roll-mode about the Torque-Roll-Axis. Response of the CG in other co-ordinates will be identically zero. They also derived analytical expressions for optimal parameters in the special case when the mounting planes are fixed. Albeit, very useful and insightful, this assumption may be too restrictive in a production environment. For a 4-mount system there are 36 design parameters corresponding to location, orientation angles and stiffness. This implies that one may adjust only up to six variables while holding the remaining parameters constant. In this work, instead of using the aforementioned six equations directly, the TRA decoupling procedure was cast as a multi-objective optimization problem. If the frequency response of the CG, defined in the TRA coordinate system, for all degrees-of-freedom except rotation about the torque-roll-axis, is minimized with respect to all 36 variables then the resulting system will automatically satisfy the six equations and produce a completely decoupled TRA mode. The optimization problem was first solved by a standard gradient-based method implemented in the commercial finite element software MSC/NASTRAN [3]. While setting up the optimization problem in MSC/NASTRAN, several difficulties were encountered. First, it was not possible to define location and spring rate of the mounts simultaneously as a common set of design variables in a single optimization run. Secondly, the orientation angles (euler angles) of mount could not be directly defined as design variables. An indirect method had to be developed to accomplish this. Therefore, the locations, rates and orientation had to be optimized sequentially. While this procedure produced acceptable results, the authors felt that two main problems common to all gradient-based search techniques, i) Entrapment in local minima, ii) Dependence on a good starting point, could be avoided if a nongradient based algorithm such as evolutionary computation was adapted. There are three evolutionary computation algorithms that have been successfully employed in many difficult optimization problems in industry. A long list of references on application of evolutionary computation can be found in [4]. These three independently developed paradigms: i) Genetic Algorithm (GA), ii) Evolutionary Programming (EP) and iii) Evolutionary Strategies (ES), are based on the mechanics of natural selection and natural genetics. These methods have established a reputation of being very robust search algorithms in recent years [5-9]. Genetic algorithm has been successfully applied to vehicle driveline vibration problems [10]. Here the authors compared the performance of GA with a standard simplex search algorithm. In [11], genetic algorithm was used to solve an engine mount optimization problem. In their work, the optimization problem was limited to minimization of off-diagonal terms of the stiffness matrix. This is essentially a partial elastic axis decoupling technique described in [2]. However, the authors did not compare the performance of GA with other standard search algorithms. In the present work, the Evolutionary Strategies (ES) algorithm was adapted to solve the TRA optimization problem. This particular paradigm was chosen over the other two for the following reasons: i) The algorithm is very easy to implement, ii) Linear constraint handling is straight forward and iii) It performs particularly well on continuous function optimization problems [4,6,9].

The road-profile induced low frequency vibration (less than $20 \mathrm{~Hz}$ ) felt at the steering wheel and seats by the passengers is commonly referred to as Road-Shake. Under this loading conditions, in body-on-frame sport-utilityvehicles, typically there is a lot of interaction between powertrain and suspension modes. The global body modes 
such as bending and torsion are usually well separated from the suspension and powertrain modes. A common strategy often adapted to attenuated the suspension/powertrain coupled vibration is to use the powertrain rigid body modes as a tuned-mass-absorber [12]. In particular, engineers attempt to place the bounce (vertical translation) mode frequency of the powertrain on top of the front suspension hop mode (vertical translation) frequency while the roll mode frequency of the powertrain is placed at or near the suspension tramp mode (out-of-phase vertical translation) frequency. If the powertrain rigid-body modes are reasonably decoupled then they can be effectively used as a tuned-mass-absorber for both hop and tramp mode of the suspension. This is even more effective when the suspension is not independent (solid axles).

In this work, the ideas introduced in [2] were extended and applied to a production vehicle program with a V6 powertrain supported by a 4-mount system. The basic strategy adopted here was three fold: i) Decouple powertrain rigid-body modes by TRA decoupling procedure and obtain uncoupled roll mode for idle vibration quality, ii) Lower the roll-mode for increased vibration isolation, and finally, iii) Adjust the frequencies of the powertrain modes such that they can function as a tuned-mass-absorber to attenuate suspension-resonance related system response due to rough road profile input. In addition, an envelop was defined around the nodal point of the powertrain flexible bending mode, for transmission mount locations. Finally, the lower-limit of the mount isolators and corresponding orientation angles were fixed such that the static load-bearing and vehicle handling requirements are not compromised. The performance of the optimal mount parameters were evaluated in a full-vehicle production finite element model subjected to idle torque and road-shake load cases.

The remaining part of this paper is organized as follows: 1) Derivation of the TRA decoupling scheme, 2) Formulation of the optimization problem in MSC/NASTRAN followed by ES, 3) Finite element simulation of Idle-Shake and Road-Shake, 4) Results and 5) Conclusions.

\section{Equations of motion}

The $(6 \times 6)$ equations of motion of a rigid-body powertrain $\mathrm{CG}$ in a reference co-ordinate system can be expressed in matrix form as follows:

$$
[M] \overrightarrow{\ddot{x}}_{g}+[C] \overrightarrow{\dot{x}}_{g}+[K] \vec{x}_{g}=\overrightarrow{0}
$$

The mass matrix is given by

$$
\left[\begin{array}{cccccc}
m & 0 & 0 & 0 & 0 & 0 \\
0 & m & 0 & 0 & 0 & 0 \\
0 & 0 & m & 0 & 0 & 0 \\
0 & 0 & 0 & I_{x x} & I_{x y} & I_{x z} \\
0 & 0 & 0 & I_{y x} & I_{y y} & I_{y z} \\
0 & 0 & 0 & I_{z x} & I_{z y} & I_{z z}
\end{array}\right]
$$

where $m$ is the mass of the powertrain and [I] is the corresponding inertia matrix. The diagonal isolator stiffness matrix of each mount in local co-ordinate system is transformed to the global reference co-ordinate system. Then the sub-matrices are assembled to form the global stiffness matrix $[\mathrm{K}]$. Similarly, a proportional damping matrix, [c] can also be transformed and assembled to obtain the global damping matrix [C]. Derivation and definition of the elements of global symmetric $[\mathrm{K}]$ and $[\mathrm{C}]$ matrices are given in [13-15]. The eigen value problem associated with Eq. (1) is expressed as

$$
\mathbf{K u}=\lambda \mathbf{M u}
$$

Solving Eq. (3) yields six eigen values, $\lambda$ and the corresponding modal matrix, U. Frequency response of the CG, $x_{g}(\omega)$, can be computed by the modal superposition technique [16]. The Fourier transform of the impulse response function $h_{r}(\tau)$ is defined as

$$
H_{r}(\omega)=\frac{1}{\omega_{r}^{2}-\omega^{2}+2 j \zeta_{r} \omega_{r} \omega}
$$


where $\omega_{r}$ is the $r^{t h}$ modal frequency, $\omega$ is the driving frequency, $\zeta_{r}$ is the $r^{t h}$ proportional modal damping ratio. The transfer function matrix for the system is given by

$$
[H(\omega)]_{i j}=\sum_{r=1}^{\text {nmode }} U_{i r} U_{j r} H_{r}(\omega)
$$

where $U_{i j}$ are the coefficients of the modal matrix. Finally, the response of the CG co-ordinates due to a harmonic input can be expressed in terms of the modal coefficients as follows

$$
X_{i}(\omega)=[H(\omega)] \vec{F}(\omega)
$$

where $\vec{F}(\omega)$ is a harmonic load vector. The response magnitude of the $i^{\text {th }}$ degree-of-freedom can be written as

$$
\left|X_{i}(\omega)\right|^{2}=\sum_{l=1}^{\text {ndof ndof }} \sum_{m=1}[H]_{i l}^{*}[H]_{j m} F_{l}^{*}(\omega) F_{m}(\omega)
$$

\section{TRA decoupling}

As stated in [15], when an oscillatory torque is applied to a rigid body, supported by soft isolators, about an axis other than one the principal axes of inertia, it will rotate about an apparent axis that is neither a principal axis of inertia, nor the axis of the oscillatory torque. This axis is referred to as the torque-roll-axis (TRA). Mathematical definition of TRA is given in [2]. One can define a co-ordinate system in which the x-axis is parallel to the TRA. In the following, such a co-ordinate system will be denoted by $\Gamma_{\text {TRA }}$. Let the inertial reference co-ordinate system for the rigid powertrain be denoted by $\Gamma_{g}$. Note that the crank-shaft axis is assumed to be parallel to the $x$ axis of $\Gamma_{g}$. The equations of motion given by Eq. (1) can also be expressed in the $\Gamma_{\text {TRA }}$ axis instead of the inertial co-ordinate system, $\Gamma_{g}$. Of course, now the $[M],[K]$ and $[C]$ must be transformed and reassembled in the $\Gamma_{\text {TRA }}$ system. The undamped equations of motion in Eq. (1) is redefined in $\Gamma_{\text {TRA }}$ axis as follows

$$
[M] \overrightarrow{\ddot{x}}_{\mathrm{TRA}}+[K] \vec{x}_{\mathrm{TRA}}=\overrightarrow{0}
$$

corresponding eigen value problem is

$$
\mathbf{K} \mathbf{q}_{\mathrm{TRA}}=\lambda \mathbf{M} \mathbf{q}_{\mathrm{TRA}}
$$

A complete decoupling of the TRA mode from other physical modes is possible only when the TRA direction, $\mathbf{q}_{\text {TRA }}=\left[\begin{array}{lllll}0 & 0 & 0 & 1 & 0\end{array}\right]^{T}$ is one of the natural modes. Substituting this condition in Eq. (9) produces six equations [2]

$$
\begin{aligned}
& \sum_{i=1}^{n}\left(k_{x z_{i}} a_{y_{i}}-k_{x y_{i}} a_{z_{i}}\right)=0 \\
& \sum_{i=1}^{n}\left(k_{y z_{i}} a_{y_{i}}-k_{y y_{i}} a_{z_{i}}\right)=0, \sum_{i=1}^{n}\left(k_{z z_{i}} a_{y_{i}}-k_{y z_{i}} a_{z_{i}}\right)=0, \\
& \sum_{i=1}^{n}\left(k_{y y_{i}} a_{z_{i}}^{2}+k_{z z_{i}} a_{y_{i}}^{2}-2 k_{y z_{i}} a_{y_{i}} a_{z_{i}}=\lambda I_{x x},\right. \\
& \sum_{i=1}^{n}\left(k_{x z_{i}} a_{y_{i}} a_{z_{i}}+k_{y z_{i}} a_{x_{i}} a_{z_{i}}-k_{z z_{i}} a_{x_{i}} a_{y_{i}}-k_{x y_{i}} a_{z_{i}}^{2}\right)=-\lambda I_{x y}, \\
& \left.\sum_{i=1}^{n} k_{x y_{i}} a_{y_{i}} a_{z_{i}}+k_{y z_{i}} a_{x_{i}} a_{y_{i}}-k_{y y_{i}} a_{x_{i}} a_{z_{i}}-k_{x z_{i}} a_{y_{i}}^{2}\right)=-\lambda I_{x z}
\end{aligned}
$$

In the above equations, $n$ is the number of mounts, $k_{i}$ is an element of the stiffness matrix, and $\vec{a}_{i}$ are the elements of the position vectors corresponding to the $i^{t h}$ mount, in the $\Gamma_{\text {TRA }}$ co-ordinate system. These equations represent the conditions for a complete decoupling of TRA mode. These equations may be satisfied by adjusting up to six mounting parameters while holding the remaining constant. 


\section{The optimization problem}

Suppose a set of mounting parameters is found such that all six equations in Eq. (10) are satisfied. In this case, if a unit oscillatory torque is applied about the crank-shaft axis, then the frequency response of the CG in $\Gamma$ TRA will all go to zero except in the TRA degree-of-freedom with a single peak corresponding to the roll mode of the powertrain. This implies that if the CG frequency response, of all degrees-of-freedom except the roll direction in $\Gamma_{\text {TRA }}$ co-ordinate system (Dof \#4), are minimized with respect to the mounting parameters then Eq. (10) would be automatically satisfied and the system will approach complete TRA decoupling. In other words, the TRA decoupling procedure can be formulated as an optimization problem as follows

$$
\begin{aligned}
\frac{\partial}{\partial p_{j}} \operatorname{Max}\left(\left|X_{\operatorname{TRA}_{i}}(\omega, \vec{p})\right|^{2}\right)= & \frac{\partial}{\partial p_{j}} \operatorname{Max}\left(\sum_{l=1}^{n \text { dof }} \sum_{m=1}^{\text {ndof }}[H]_{i l}^{*}[H]_{j m} F_{l}^{*}(\omega) F_{m}(\omega)\right)=0 \\
& i=1, \ldots, 6 \quad i \neq 4 \quad j=1,2, \ldots, N
\end{aligned}
$$

where $i$ is the CG response degree-of-freedom, $p_{j}$ are the mounting parameters $j=1, \ldots, N$. Equation (11) will be satisfied only when the coefficients of the modal matrix $\left[U_{\mathrm{TRA}}\right]$ obtained from Eq. (9) take appropriate values such that Eq. (10) are satisfied. A multi-objective MIN-MAX problem in Eq. (11), can be combined so that one needs to minimize only a global scalar objective function in the following manner $[17,18]$

$$
\begin{aligned}
& \text { Minimize } f\left(\beta_{1}, \beta_{2}, \ldots \beta_{6}\right)=\sum_{i=1}^{6} w_{i} \beta_{i} \quad i \neq 4 \\
& \text { subjected to } \frac{R_{i}(\vec{p})}{R_{\max _{i}}}-\beta_{i}+1 \leqslant 1, \quad 0 \leqslant \beta_{i} \leqslant 1 \\
& \text { and } p_{j}^{L} \leqslant p_{j} \leqslant p_{j}^{U} j=1, \ldots, N
\end{aligned}
$$

In Eq. (12), $f\left(\beta_{i}\right)$ is the global objective function, $\beta_{i}$ 's are auxiliary variables and $w_{i}$ 's are the corresponding weighting constants. In Eq. (13), $R_{i}$ are the peak values of the CG frequency response functions, $R_{i}=\operatorname{Max}\left(\left|X_{\mathrm{TRA}_{i}}(\omega)\right|\right)$. The $R_{\max _{i}}$ values are normalization constants set at the beginning of the optimization problem such that $R_{i} / R_{\max _{i}} \leqslant 1$. When the optimizer tries to minimize $f\left(\beta_{i}\right)$ by letting $\beta_{i} \rightarrow 0$, the constraints in Eq. (13) are violated. In order to satisfy the constraints, the optimizer must perturb the design parameters, $p_{j}$ 's, so that $R_{i}(\vec{p}) \rightarrow 0$. This minimization process essentially pushes a horizontal ceiling down on the maximum peak of the frequency response functions. The weighting constants, $w_{i}$ 's, in the global objective function, provide a mechanism to emphasize on some response quantities over others. Note that Eq. (13) is a non-linear constraint since it involves the frequency response functions themselves, while Eq. (14) is a linear constraint that defines the design space.

\subsection{Gradient-based optimization}

In large-scale noise and vibration optimization problems, such as, tactile or acoustic frequency response minimization, hundreds of design parameters are often involved. In these problems, non-linear inequality constraints are quite common. Under these circumstances, gradient based optimization routines, implemented in commercial software, can efficiently compute optimal solutions, although not necessarily the global minimum. Given the size and complexity of these problems, these solutions are often quite satisfactory in an industrial environment. If the objective function is not smooth and multi-modal then with a gradient based algorithm, the best one can hope to find, is a local minimum. The choice of initial design parameter values also have a major impact on the quality of the solution. To make things worse, if the initial point in the design space corresponds to a "plateau" in the response surface then the gradient of the objective function will be very small. In this situation, it is often very difficult to get the optimizer "started". The only recourse is to intentionally choose a starting point that violates the constraints or change the perturbation parameters of the algorithm, otherwise the optimizer will stall within a few cycles of iteration. TRA decoupling, on the other hand, is a relatively simple problem involving only a few parameters (27 


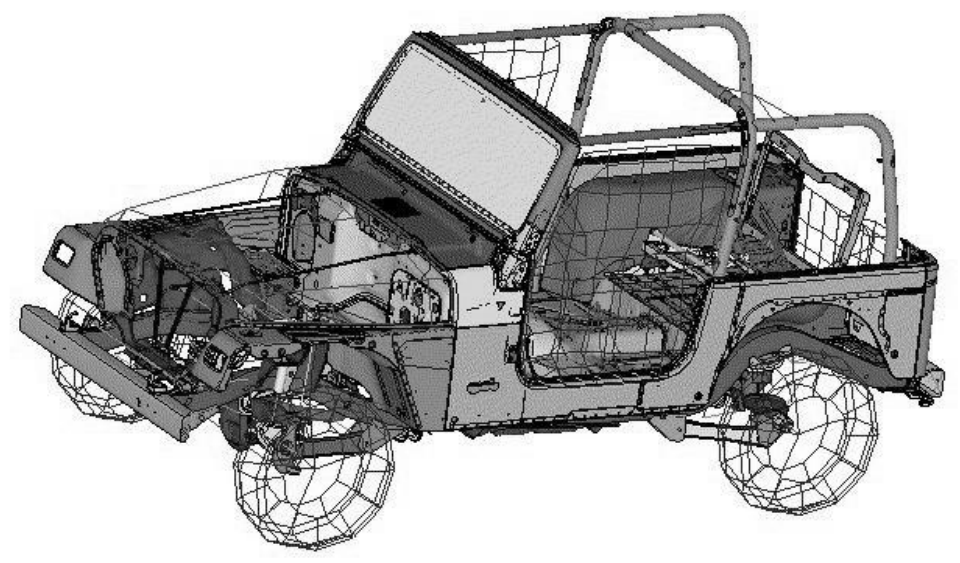

Fig. 1. Full system finite element model of a 4WD Sport Utility Vehicle.

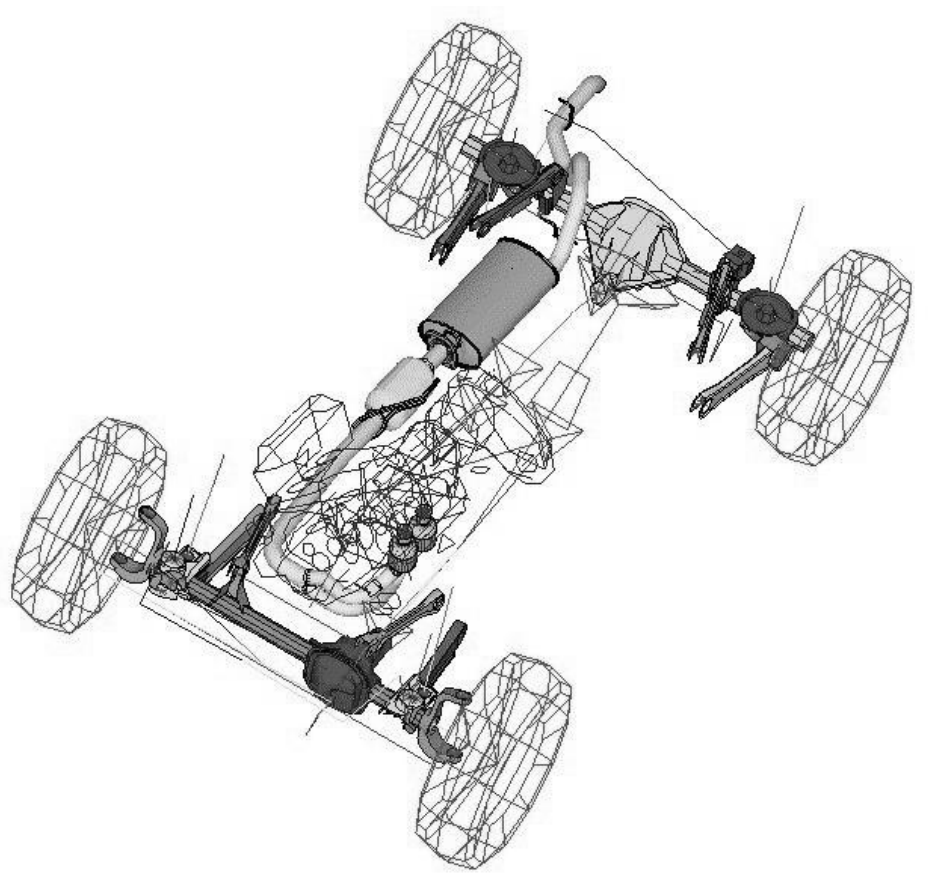

Fig. 2. Driveline, powertrain, and exhaust system of a 4WD Sport Utility Vehicle.

for 3-mount and 36 for 4-mount systems). The computation cost of function evaluation, is also minimal in this case. Therefore, a stochastic search algorithm, such as, evolutionary computation is expected to be far more efficient and effective in finding the global minimum for this problem.

In this work, the TRA optimization problem defined by Eqs (12)-(14) was solved using the optimization module of the commercial finite element software, MSC/NASTRAN. The default algorithm of the module, "Modified Method of Feasible Directions" [3,19], was used in all MSC/NASTRAN runs. Due to software implementation issues, it was not possible to include both, the locations and the stiffness values of the mounts, in the same optimization run. Moreover, the software did not provide any direct way of including the euler angles (orientation) of the mounts as design variables. To circumvent the problem, an alternate approach, explained in the following, was developed. The stiffness matrix of a given mount in the local coordinate system is transformed by the finite element solver before 


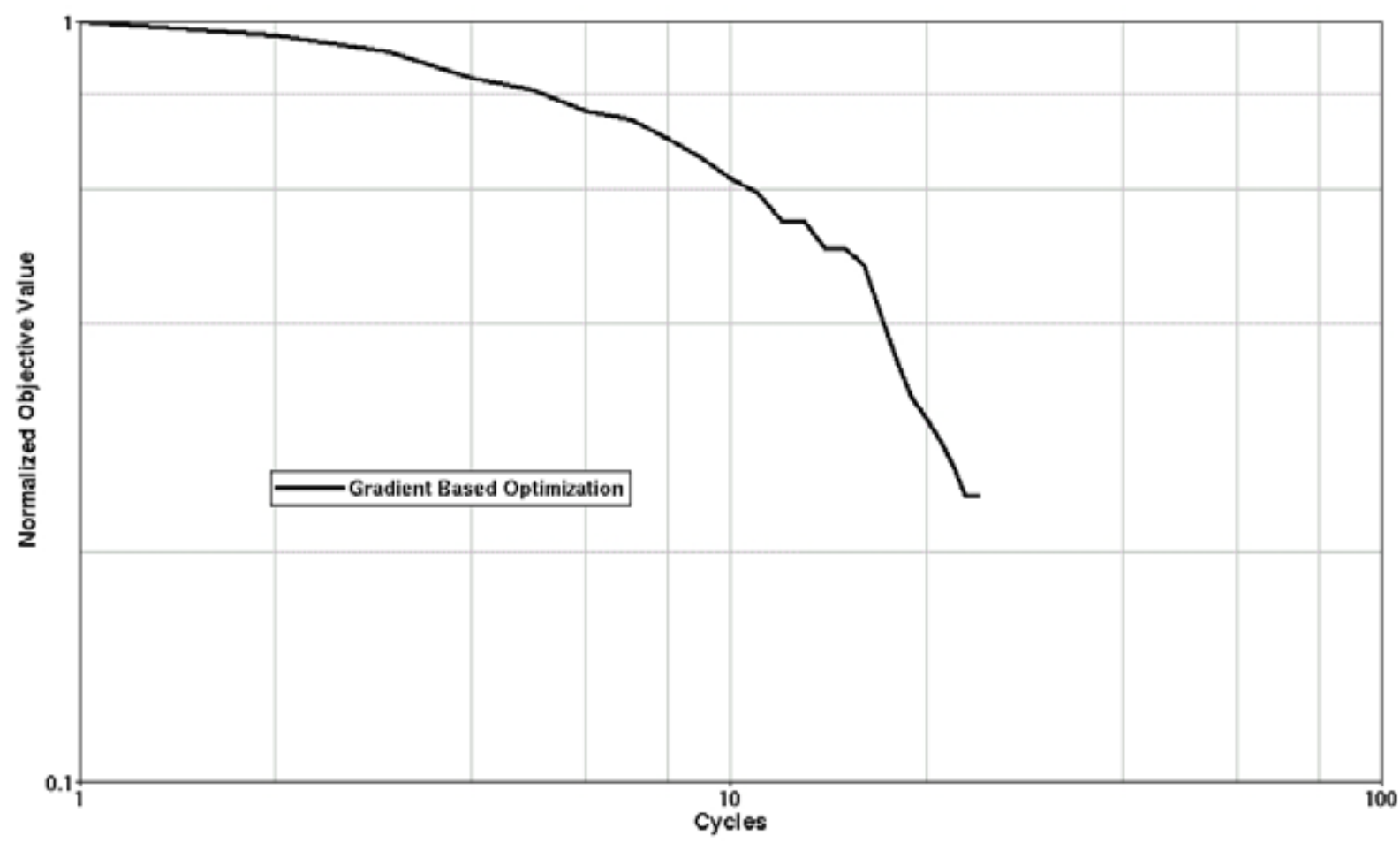

Fig. 3. Gradient based optimization performed by MSC/NASTRAN. Normalized objective function values are plotted against number of optimization cycles. Hard convergence was obtained in 25 cycles.

assembly of the global $[K]$ matrix. The transformation matrix can be expressed in terms of the euler angles, $\alpha, \beta$, and $\gamma$, as follows

$$
\begin{aligned}
& {\left[R_{\alpha}\right]=\left[\begin{array}{ccc}
1 & 0 & 0 \\
0 & \cos (\alpha) & \sin (\alpha) \\
0 & -\sin (\alpha) & \cos (\alpha)
\end{array}\right]} \\
& {\left[R_{\beta}\right]=\left[\begin{array}{ccc}
\cos (\beta) & 0 & -\sin (\beta) \\
0 & 1 & 0 \\
\sin (\beta) & 0 & \cos (\beta)
\end{array}\right]} \\
& {\left[R_{\gamma}\right]=\left[\begin{array}{ccc}
\cos (\gamma) & -\sin (\gamma) & 0 \\
\sin (\gamma) & \cos (\gamma) & 0 \\
0 & 0 & 1
\end{array}\right]}
\end{aligned}
$$

If a 3-2-1 rotation sequence is assumed then the transformation matrix can be defined as $[T]=\left[R_{\gamma}\right]\left[R_{\beta}\right]\left[R_{\alpha}\right]$. The stiffness of a mount transformed to global co-ordinate system can be expressed as $\left[k_{g}\right]=[T]^{T}[k][T]$. One can now add the euler angles as auxiliary design variables for each mount and then define the transformation equations relating the mount stiffness values to the euler angles in MSC/NASTRAN. Instead of rotating the actual coordinate system, one simply subjects the local mount stiffness matrix to a "virtual rotation". Since a rotation of the local $[k]$ matrix will result in non-zero off-diagonal terms, these must also be included as design variables. The concept of "virtual rotation" had to be implemented as a separate optimization run for euler angles because mount stiffness values themselves were being used to update the euler angles. In summary, optimization of location, stiffness and orientation were executed sequentially. 

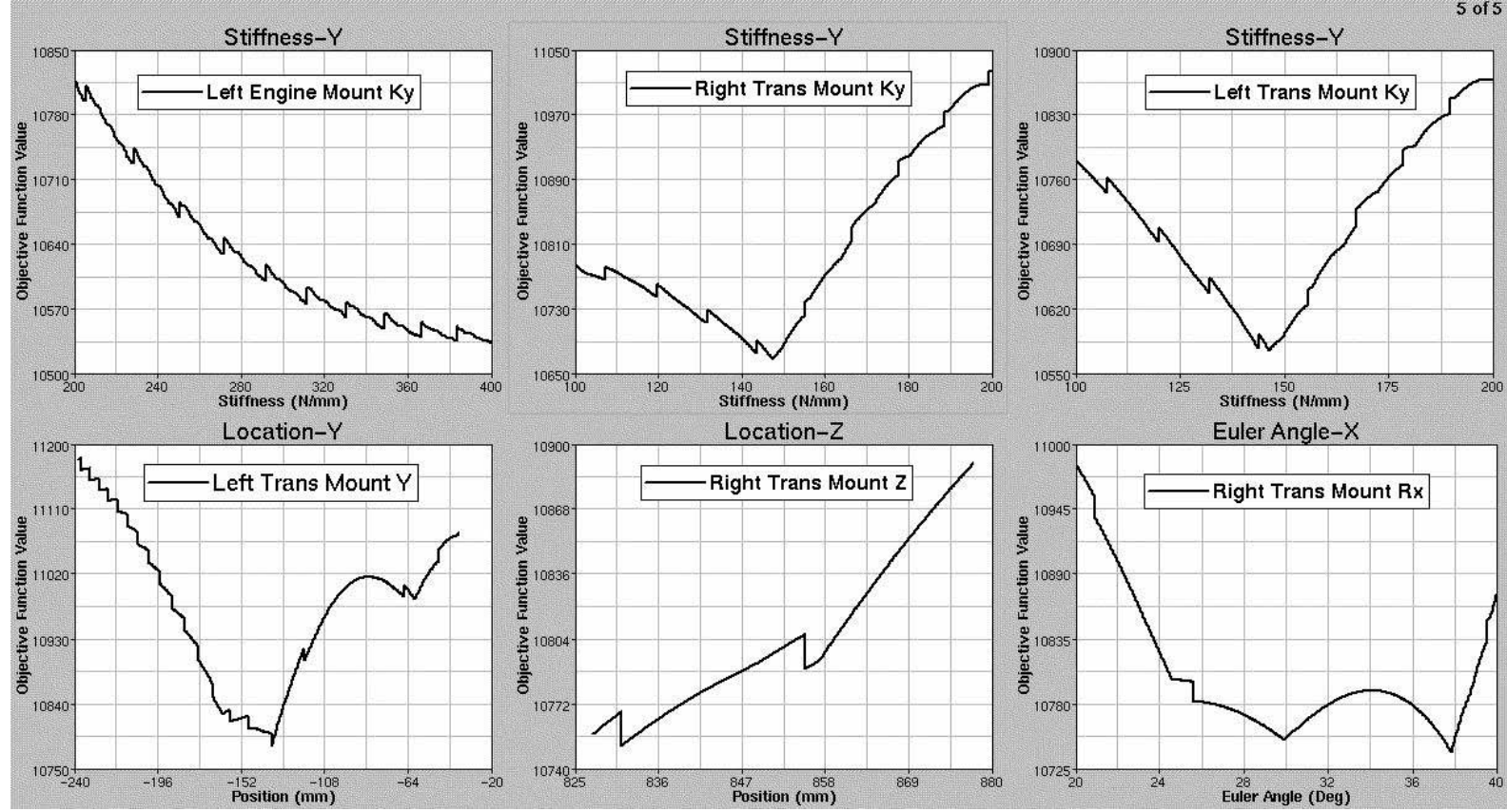

Fig. 4. In this figure, the non-linear, discontinuos and multi-modal nature of the response surface is quite evident.

\subsection{Evolutionary computation}

If unlimited computer resources are available, one can always perform a stochastic search in the design space to find the elusive global minimum. Such an approach is impractical even for very simple objective functions due to the size of the search space. Among many, one of the widely used stochastic search algorithms is Simulated Annealing [7]. The algorithm is mathematically guaranteed to converge at some point in time and it is less likely to get stuck in local minima. The algorithm, like gradient based methods, iterates towards a single best solution. However, a very slow rate of convergence, and difficulty in choosing appropriate values for the control parameters, this algorithm appears less attractive in many applications. Evolutionary computation, in contrast, maintains a population of potential solutions. Inspired by the principles of natural evolution and inheritance, it applies some selection process based on the "fitness" of "individuals" and some "genetic" operators. All algorithms belonging to this class have the following features. An evolutionary algorithm is a probabilistic method which maintains a population of solution vectors known as "individuals" for each iteration. Each solution or individual is evaluated to give a measure of it's "fitness". Usually, fitness implies the value of the objective function in some form. Then a new population is formed by selecting the more fit solution vectors. The Members of the new population are then transformed by means of "genetic" operators to form an all new population of solutions. Commonly used operators are recombination, mutation, cross-over etc. The selection process can be stochastic or deterministic. Three most widely used algorithms that fall into the category of evolutionary computation are i) Genetic Algorithm (GA), ii) Evolutionary Programming (EP) and iii) Evolutionary Strategies (ES). These algorithms differ in the type of genetic operators used, the selection methods used, how constraints are handled, and finally, how the solution vector is represented (binary or floating point numbers). An overview of the most common instances of these algorithms is given in [4]. More advanced selection, mutation and other operators along with constraint handling techniques are discussed in [20].

Compared to Genetic Algorithms and Evolutionary Programming, in their standard form, Evolutionary Strategies has been shown to perform very well on multi-modal continuous function optimization problems [9]. The algorithm, in its standard form, is very easy to implement. It also provides a simple way of handling inequality constraints [8]. The algorithm stochastically samples the design space to form an initial population of solution vectors. It then creates a larger population of offspring solution vectors by applying recombination and mutation operators on the parent 


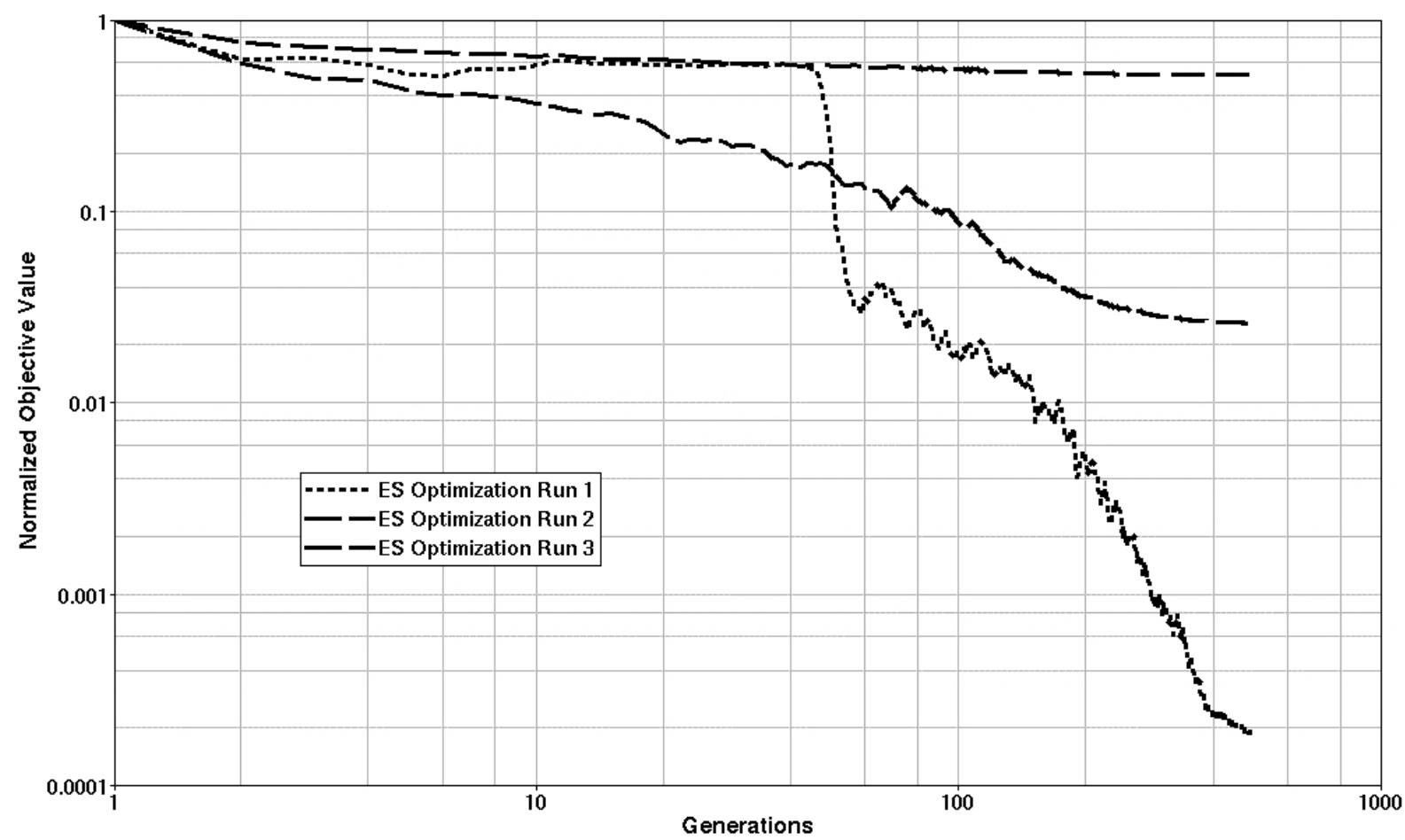

Fig. 5. Normalized objective function values are plotted against number of generations in ES optimization. Three different convergence patterns are shown here.

population. Finally, it deterministically selects the best performing individuals to form the new parent population. This evolutionary process is continued over many generations or cycles until some convergence criterion is met. During the entire process, the parent to offspring population ratio is held fixed. The mutation parameters are initially set to some default value. The parameters evolve or self-adapt on-line during the optimization process and require no user intervention. Only two fixed control parameters (known as learning parameters) have to be defined by the user. A brief description of ES in its standard form implemented in this study is in order. The algorithm has three basic steps for generating offspring from parents: i) recombination, ii) mutation and iii) selection. The elements of a parent solution vector and the corresponding mutation parameters are evolved in the following manner

$$
\begin{aligned}
\sigma_{i}^{\prime} & =\sigma_{i} \cdot \exp \left(\tau^{\prime} \cdot N(0,1)+\tau \cdot N_{i}(0,1)\right) \\
\tau & =\frac{1}{\sqrt{2 \sqrt{n}}} ; \tau^{\prime}=\frac{1}{\sqrt{2 n}} \\
x_{i}^{\prime} & =x_{i}+\sigma_{i}^{\prime} \cdot N_{i}(0,1)
\end{aligned}
$$

where $n$ is the number of design variables, $\sigma_{i}$ is the current mutation parameter for the $i^{t h}$ element mutated to $\sigma_{i}^{\prime}$, $\tau_{i}^{\prime}$ and $\tau_{i}$ are the learning parameters, $N(0,1)$ is a standard normal random number sampled once for a given individual solution vector, $N_{i}(0,1)$ is a standard normal random number sampled separately for each element, and $x_{i}^{\prime}$ is an element of the new solution vector mutated from $x_{i}$. Recombination occurs at two levels: the mutation parameters are subjected to panmictic-intermediate recombination while the elements of the solution vector are subjected to discrete recombination [9]. The purpose of recombination is to exchange "genetic" information between two parent solution vectors for producing offsprings. This is accomplished by randomly choosing two parents from the population first. Then a given element of the offspring vector is obtained by randomly selecting the corresponding element from one of the two parents. The mutation parameters of the offspring vector are obtained by averaging the corresponding mutation parameters from two randomly chosen parents for each element. The selection process implemented here is known as the "comma" strategy, $(\mu, \lambda)$. Suppose $\mu$ parents are recombined and mutated to produce $\lambda$ offsprings 


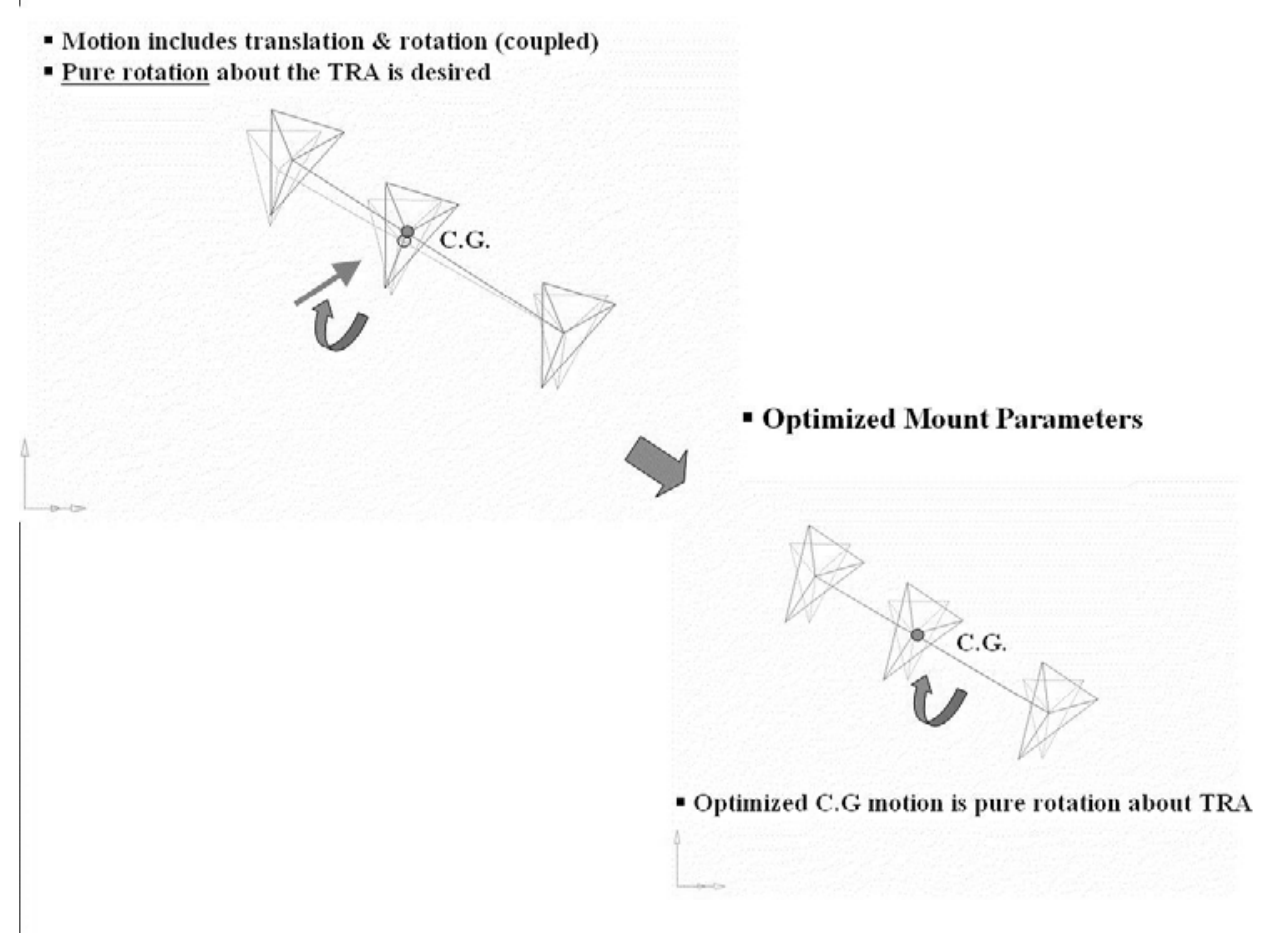

Fig. 6. The optimal CG motion due to unit torque about the crank-shaft axis, compared with that of the baseline configuration, is shown here.

$(\lambda>\mu)$. Then according to $(\mu, \lambda)$ strategy, only the best performing $\mu$ offsprings are retained as parents for the next generation and the rest are eliminated. The parent to offspring population ratio is fixed at 1:7. Inequality constraints can be handled by simply repeating the creation of an individual solution vector until all constraints are satisfied and then the new individual is accepted. The ES algorithm population parameters used in this study are as follows: $\mu=15$ and $\lambda=100$.

\subsection{Implementation of evolutionary strategies}

Evaluation of the inequality constraints in Eq. (13) requires one to solve the eigen value problem in Eq. (9) and then compute the frequency response functions given by Eq. (7). The simple constraint handling technique described in the previous section, in this case, would be very inefficient due to the computation overhead of repeatedly solving Eqs (7) and (9). Fortunately, the inequalities in Eq. (13) can be easily eliminated by a slight modification of the objective function as follows

$$
\begin{aligned}
& \text { Minimize } f\left(\beta_{1}, \beta_{2}, \ldots \beta_{6}\right)=\sum_{i=1}^{6} w_{i} \beta_{i} \quad i \neq 4 \\
& \text { where } \beta_{i}=\frac{\operatorname{Max}\left(\left|X_{\mathrm{TRA}_{i}}(\omega, \vec{p})\right|\right)}{X_{i}^{\operatorname{Ref}}} \\
& \text { subjected to } p_{j}^{L} \leqslant p_{j} \leqslant p_{j}^{U} \quad j=1, \ldots, N
\end{aligned}
$$

The auxiliary variables, $\beta_{i}$, in Eq. (12) have been eliminated and redefined simply as the normalized frequency response peak values. In the above, $X_{i}^{\text {Ref }}$ are normalization constants, $w_{i}$ 's are the weighting constants and $p_{j}$ 's are the mounting parameters. By solving the optimization problem in Eq. (12) or Eq. (17) one could obtain a TRA decoupled roll mode of the powertrain. But the user has no control on where that roll mode will appear in the frequency axis. One of the objectives of this paper was to slide the roll mode down the frequency axis as much 


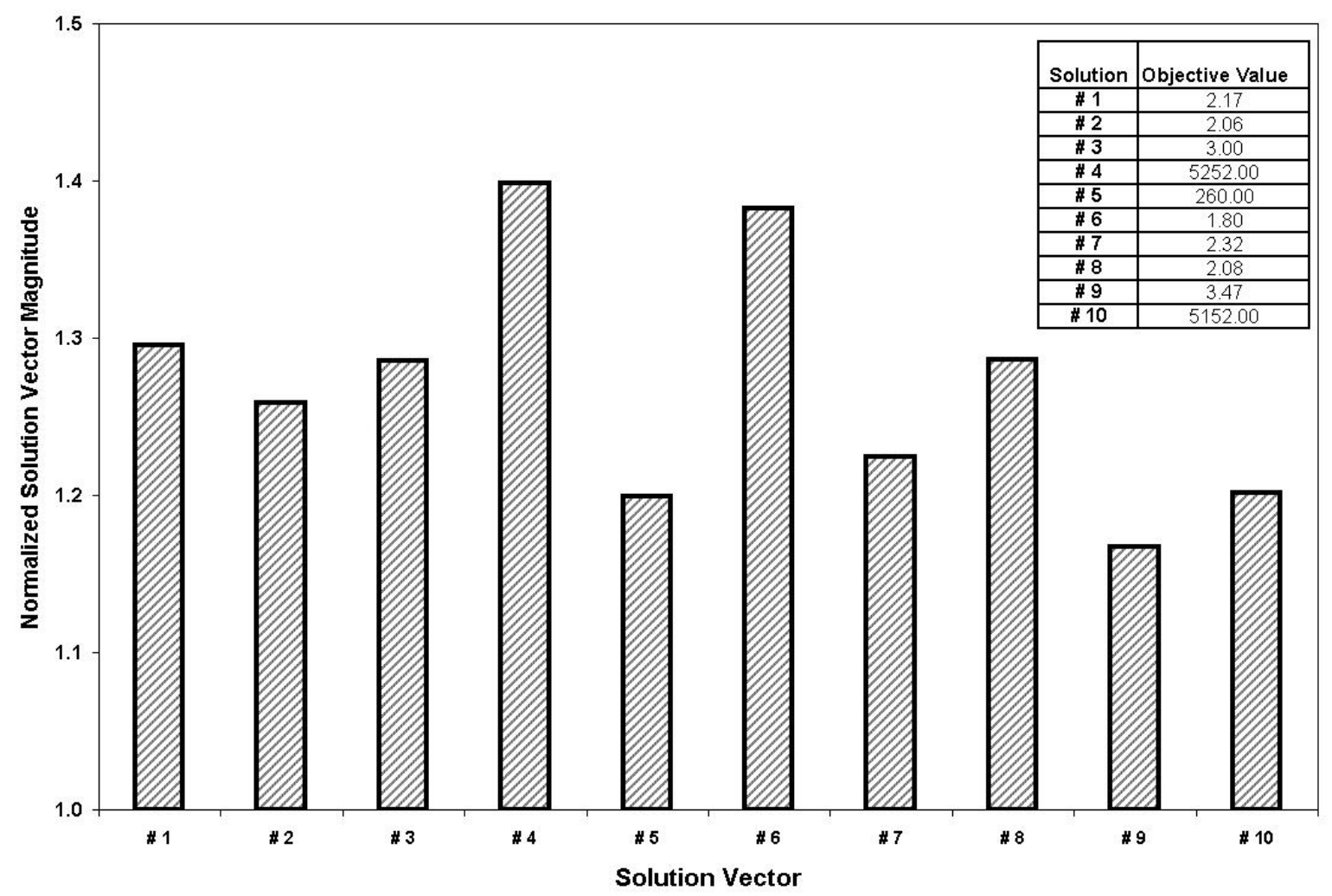

Fig. 7. Magnitude of solution vectors, normalized with respect to that of the baseline, for ten different ES optimization runs are shown here. The corresponding final objective function values are also shown in the table.

as possible for maximum isolation. Another objective was to deliberately place the roll mode at or near the front suspension tramp mode. Neither of these two objectives can be pursued if there is no control over the roll mode frequency. Complete decoupling conditions given by Eq. (10), shows that once a decoupled roll mode is obtained, one can always scale the global $[K]$ matrix by a constant factor $\alpha$, to adjust the roll mode frequency without disturbing the decoupling. However, scaling may not be possible in many situations as it might force some of the mount stiffness values below the production feasible lower limits. Therefore, Eq. (18) needs to be modified in some fashion to include this additional constraint. One way of enforcing such a constraint is to add a static penalty function [20] to the current objective function in the following manner

$$
\begin{aligned}
& \text { Minimize } f\left(\beta_{1}, \beta_{2}, \ldots \beta_{6}, \beta_{7}\right)=\sum_{i=1}^{7} w_{i} \beta_{i} i \neq 4 \\
& \text { where } \beta_{7}=\frac{f_{\text {Roll }}}{f_{\text {Roll }}^{\text {Target }}} \\
& \text { subjected to } p_{j}^{L} \leqslant p_{j} \leqslant p_{j}^{U} j=1, \ldots, N
\end{aligned}
$$

where $f_{\text {Roll }}$ is identified by locating the roll mode frequency corresponding to the peak of $\left|X_{\text {TRA }_{i}}(\omega)\right|$ in the roll degree-of-freedom ( $\operatorname{dof} i=4), f_{\text {Roll }}^{\text {Target }}$ is the target roll mode frequency. Note that the weighting constant, $w_{7}$ is set to zero when the roll mode frequency becomes less than the target frequency

$$
w_{7}=\left\{\begin{array}{l}
C, f_{\text {Roll }} \geqslant f_{\text {Roll }}^{\text {Target }} \\
0, \quad \text { otherwise }
\end{array}\right.
$$



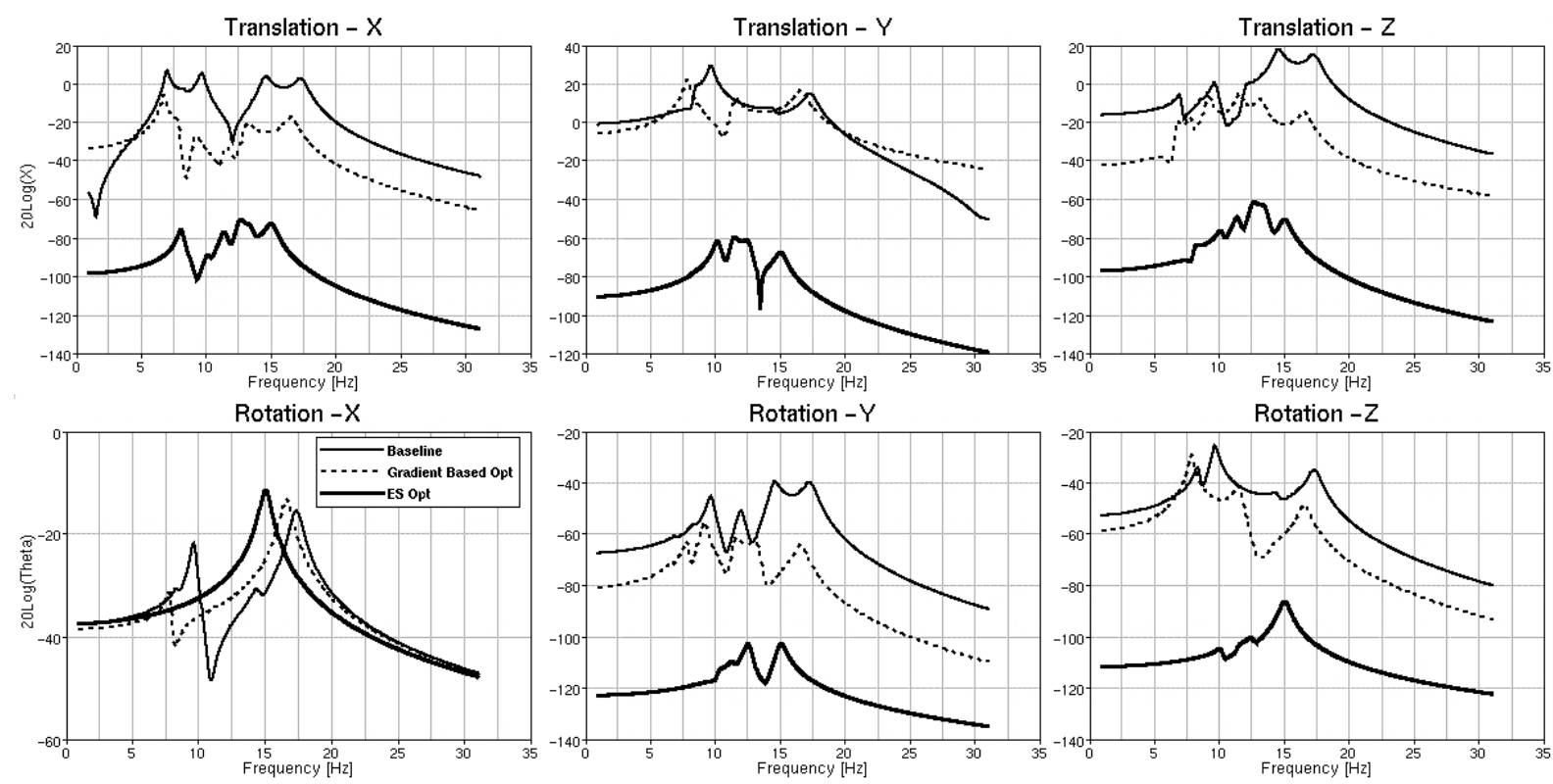

Fig. 8. CG response of the rigid powertrain by itself in TRA co-ordinate system due to a unit torque about the crank-shaft axis. The ES optimized roll-mode response (Rotation-X) is completely decoupled.

where $C$ is a weighting constant. The ES algorithm described in the previous section was implemented in a standalone $C^{++}$program. The eigen-value problem in Eq. (9) was solved by Choleski Decomposition and Jacobi Iteration techniques $[21,22]$. The frequency response functions were computed by mode superposition as shown in Eq. (7).

\section{The full-vehicle FE model}

In order to evaluate the effect of TRA decoupling on Idle and Road Shake response, a full-vehicle production finite element model of a 4WD SUV was exercised. A typical system model, as shown in Fig. 1, is in the order of million degrees of freedom. The exhaust system, axles, suspension links and other driveline components are modeled as flexible bodies. The seats and closure systems are imported into the model as CMS (Component-Mode-Synthesis) sub-structures [23]. The powertrain is modeled as a rigid body with appropriate mass and inertia. The tires and the rims are included in the system as modal models as received from the tire supplier. A typical finite element representation of the driveline, chassis, exhaust and suspension system is shown in Fig. 2. Idle shake is simulated by subjecting the powertrain to a torque spectrum appropriate for the engine type (e.g., V6, V8 etc.). A roughroad-profile wave-number spectrum is converted to a frequency spectrum for a specific speed. The tire-patch points are subjected to the road-profile frequency spectra as enforced motion. Four standard subcases are monitored for road-shake: i) Front wheels in-phase (FWIP) input, ii) Front wheels out-of-phase (FWOP), iii) Rear wheels in-phase (RWIP) and iv) Rear wheels out-of-phase (RWOP). These load-cases essentially bound the response of the vehicle for any phase relationship between the wheels for a given axle and a corresponding road profile. In both, idle and road-shake load-cases, responses are monitored at the steering column and driver's seat bolt locations.

\section{Results}

\subsection{TRA decupling optimization}

The TRA optimization problem defined by Eqs (12)-(14) was solved by using the optimization module of MSC/NASTRAN. The default algorithm of the module, "Modified Method of Feasible Directions" [3,19], was used 


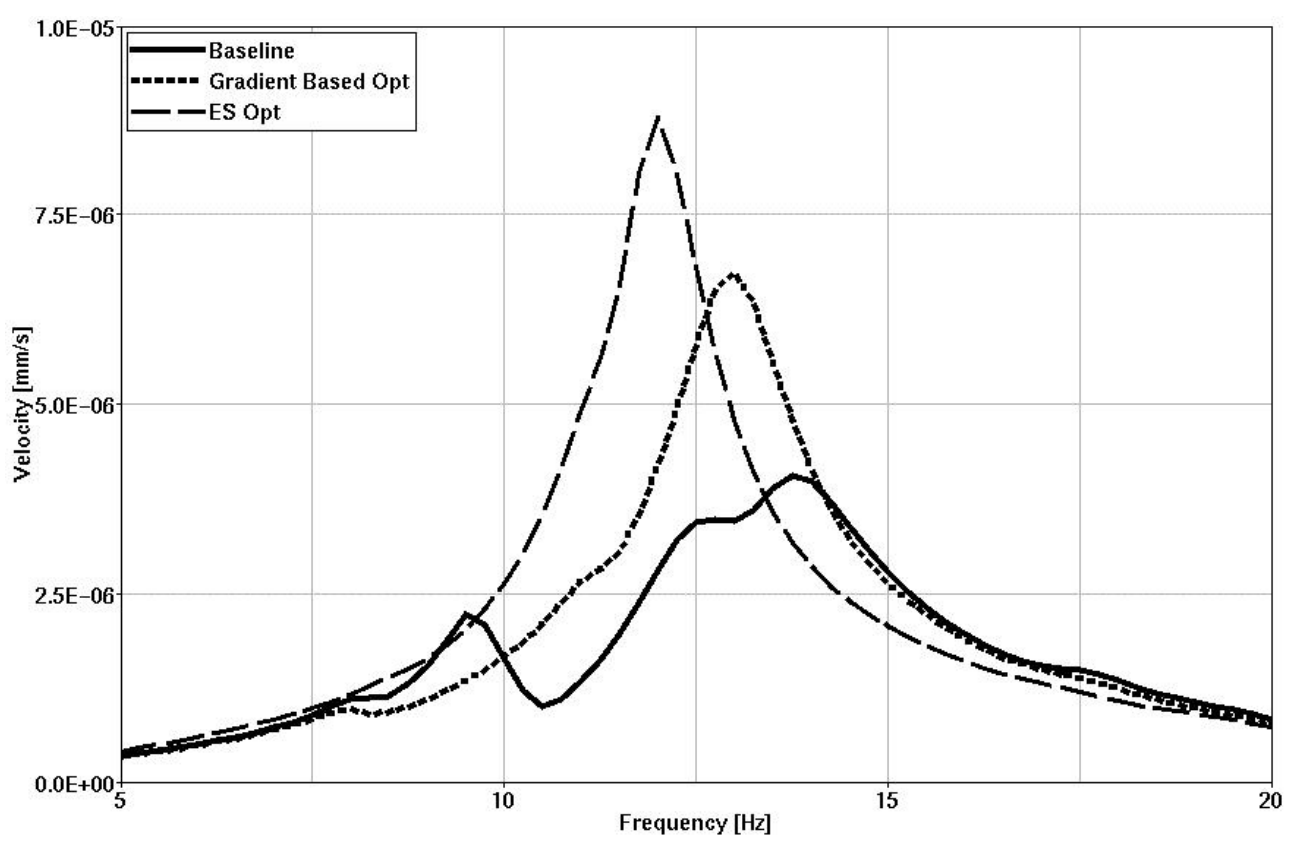

Fig. 9. CG response of the powertrain in the full-vehicle FE model, due to a unit torque about the crank-shaft axis.

\section{Baseline Mount Location}

ES Optimal Mount Location

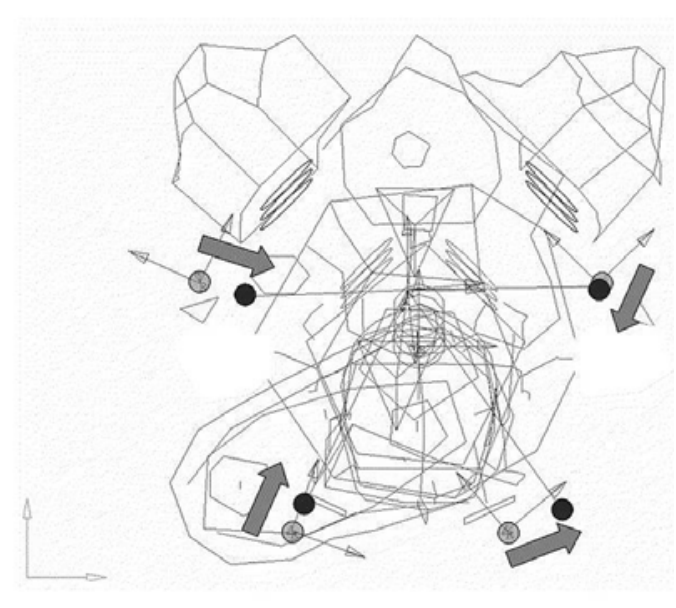

Rear View of V6 P/T

Fig. 10. ES optimized mount locations are compared with that of the baseline system.

in all MSC/NASTRAN runs. In this work, all MSC/NASTRAN optimization runs are referred to as GradientBased-Optimization. Mount location, stiffness and euler angle optimization was performed sequentially by solving Eqs (12)-(13). Production feasible bounds were imposed on all 36 mounting parameters in Eq. (14). In Fig. 3, the normalized values of the objective function are shown as a function of optimization cycles. Hard convergence was achieved in 25 cycles. Evolutionary Strategies (ES) was used to solve the optimization problem in Eq. (18). The parameter bounds were exactly the same as that of MSC/NASTRAN optimization runs. Since the response surface of the objective function cannot be visualized in the full design space, one alternative is to vary one variable at a time and 


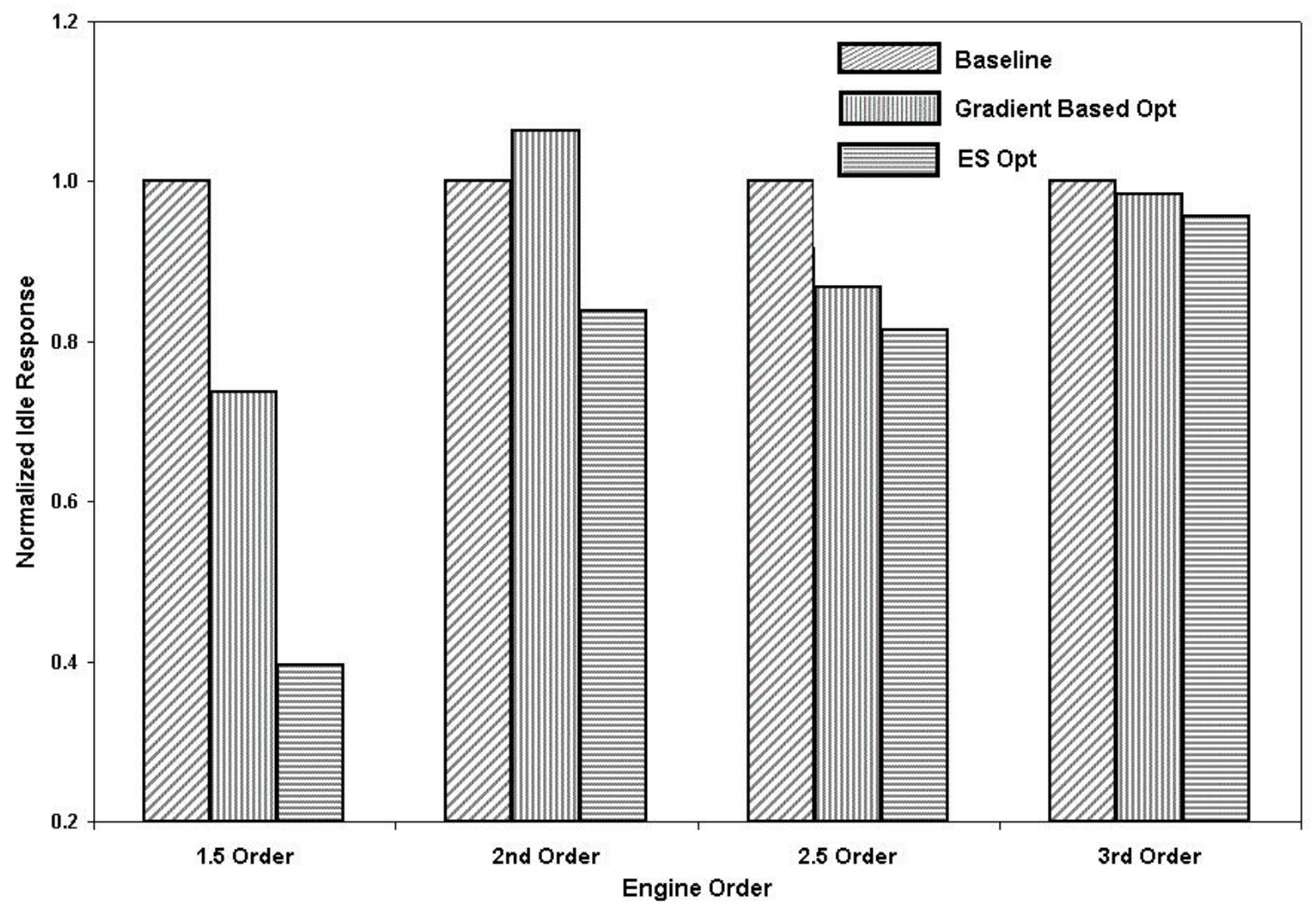

Fig. 11. Idle shake response at the steering column in the full-vehicle system for different engine orders.

examine the cross section of the response surface. In Fig. 4, the objective function response surfaces corresponding to Eq. (18), as a function of some of the design varibles is shown. In this figure, the non-linear, discontinuos and multi-modal nature of the response surface is quite evident. It is a common practice to run probabilistic search algorithms a number of times to capture as many local minima and possibly the global minimum of the objective function. In this study, ES was run with exactly the same constraints ten times. All optimization runs were limited to 500 generations. In these runs, ES exhibited different convergence patterns, most likely related to relative minima in the response surface of the objective function. Normalized objective function values for three different ES runs, capturing some of these convergence patterns, as a function of number of generations, are shown in Fig. 5. The optimal CG motion due to unit torque about the crank-shaft axis is compared with that of the baseline configuration in Fig. 6. Note that due to the additional constraint imposed on the roll mode frequency by adding a penalty function, one can not directly compare the objective function values from ES with that of MSC/NASTRAN. In Fig. 5, it appears that ES found two local and the global minimum. These convergence patterns show that the objective function is indeed multi-modal. To graphically compare the scatter of the solution vectors in design space, first the design variables, $p_{j}^{L} \leqslant p_{j} \leqslant p_{j}^{U}$, were linearly scaled [24] between $(-1,1)$ according to the following equations

$$
\begin{aligned}
& p_{j}^{L} \leqslant p_{j} \leqslant p_{j}^{U} \\
& A_{\max }=1.0, A_{\min }=-1.0 \\
& R_{j}=\frac{A_{\max }-A_{\min }}{p_{j}^{U}-p_{j}^{L}} \\
& p_{j}^{\prime}=R_{i} p_{j}+\left[A_{\min }-R_{j} p_{j}^{L}\right] \\
& -1.0 \leqslant p_{j}^{\prime} \leqslant 1.0
\end{aligned}
$$




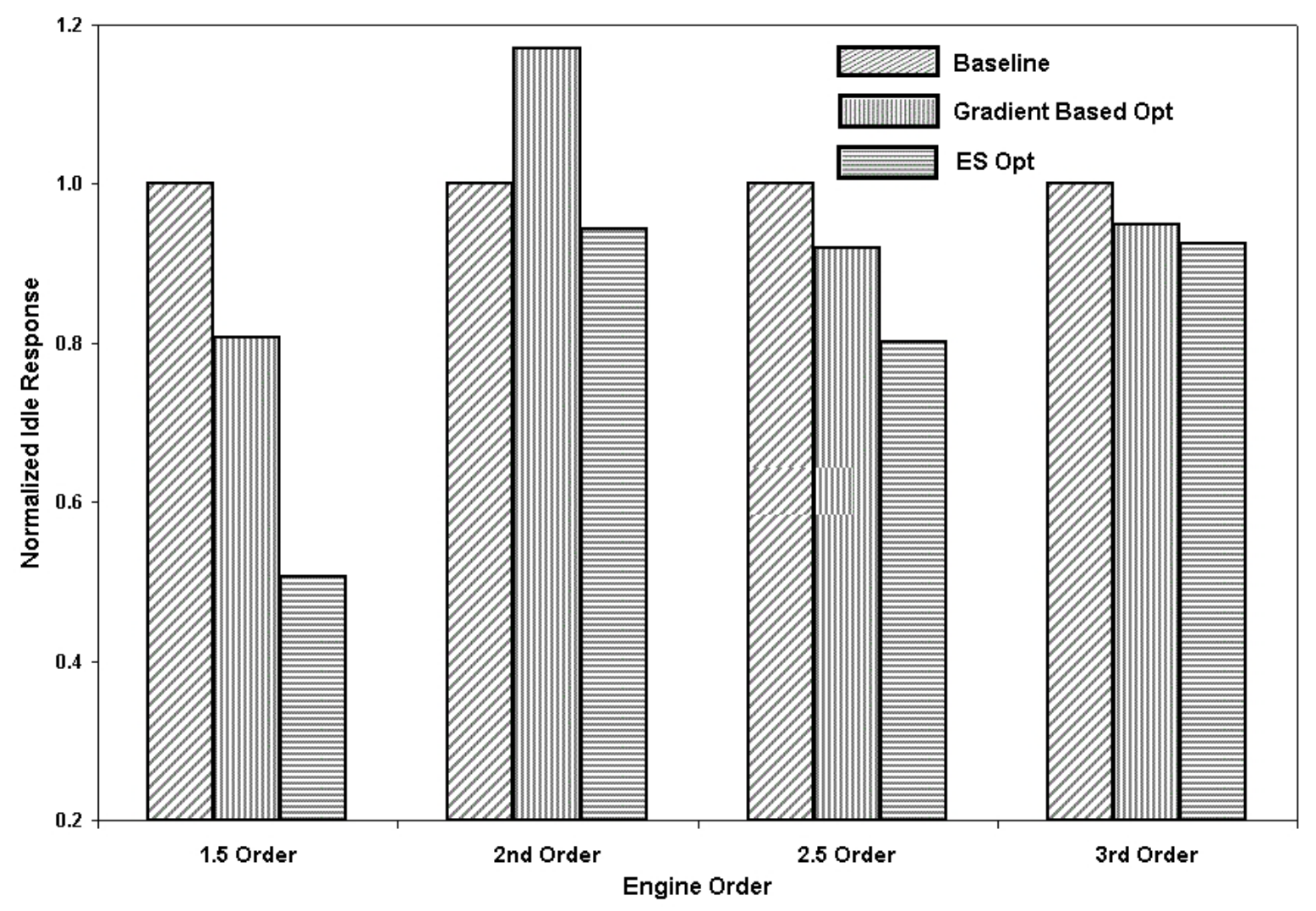

Fig. 12. Idle shake response at the driver's seat bolt location in the full-vehicle system different engine orders.

The magnitude of solution vectors, $P=\sqrt{\sum_{i=1}^{N} p_{j}^{2}}$, were then normalized with respect to that of the baseline location in design space. In Fig. 7, these magnitudes and the corresponding best objective function values from the ten runs are compared. From this plot one can conclude that the solutions obtained by ES represent different locations in the design space. In other words, potentially, multiple local minima have been identified by ES. In order to capture all the relative minima, in short of running the algorithm $\mathrm{N}$ number of times, one must resort to a more sophisticated form of the ES algorithm. Different techniques have been developed over the years to accomplish this, for example, The Sequential Niche Technique, or The Species Conserving GA, to name a few [25,26]. These techniques were not implemented in this study. Note that the quality of solutions obtained in all ten runs were significantly better than that of the gradient based optimization. In order to assess the quality of the solutions, responses at two levels were monitored: i) CG response of the rigid powertrain by itself in TRA co-ordinate system and in the full-vehicle FE model due to a unit torque spectrum applied about the crank-shaft axis, ii) Steering column and Seat response due engine idle torque and road-shake input. The CG response of the rigid powertrain by itself, in the TRA co-ordinate system is shown in Fig. 8. Here it can be seen that the best solution obtained from ES has done significantly better than MSC/NASTRAN in decoupling the system. The decoupled CG response, due to a unit torque about the crank, as expected, shows a higher amplitude single peak at the roll mode. Since the system is decoupled from other modes, concentration of energy is only at the roll mode, causing the amplitude to increase from that of the baseline system. This is deemed as a desirable feature because a pure roll mode of the power-train will serve as a more effective tuned absorber to reduced front suspension tramp related response peaks. The roll mode response (Rotation-X) produced by ES is completely decoupled, while the responses in other degrees-of-freedom are at least $70 \mathrm{~dB}$ below the baseline responses. The CG response in the full-vehicle FE model is shown in Fig. 9. Here again, it can be seen that the solution vector obtained from ES produced a completely decoupled CG response even at the full-vehicle system level. This is generally difficult to achieve due to strong coupling between the powertrain, driveline and the 

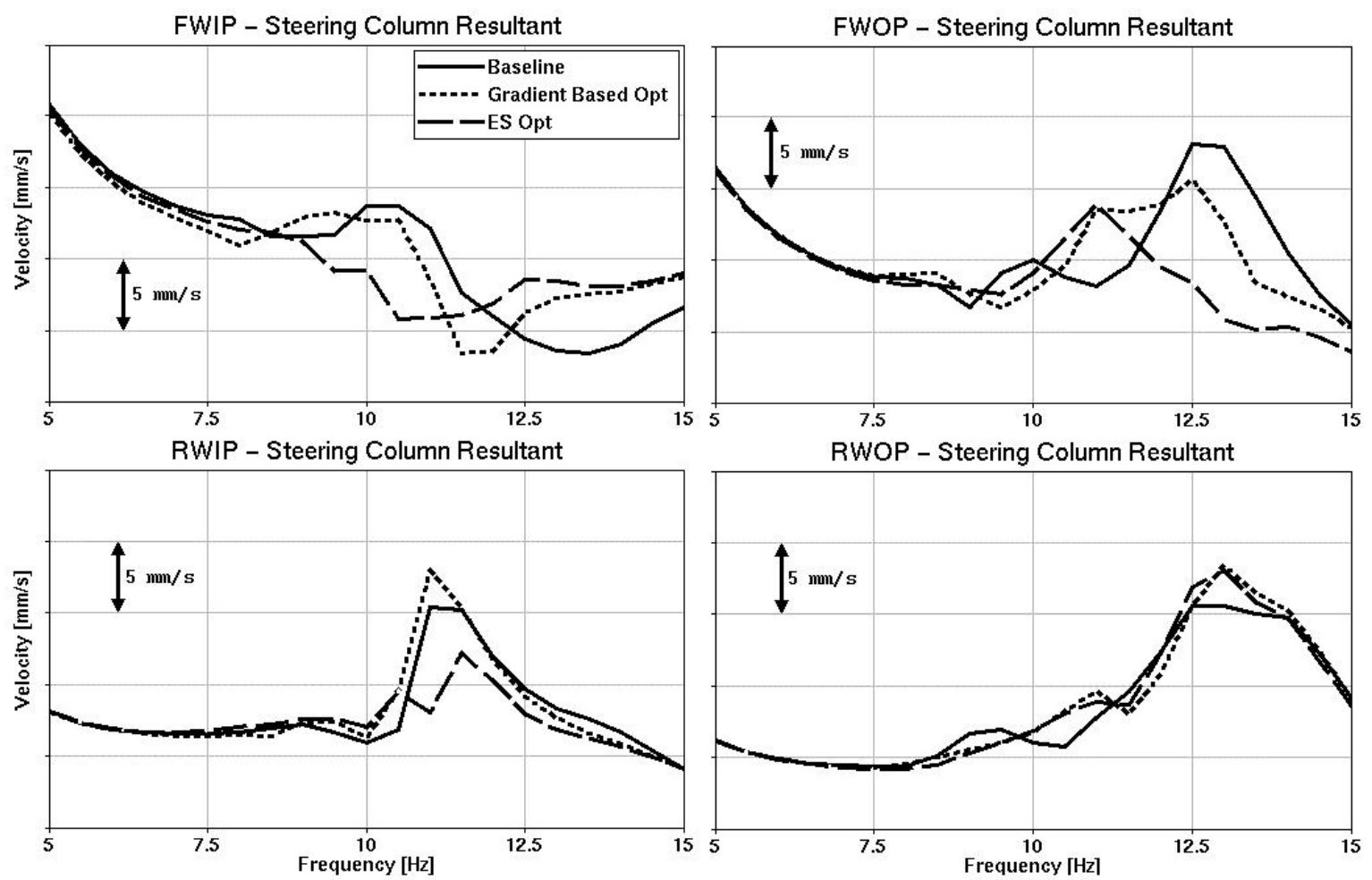

Fig. 13. Road shake response of the full-vehicle system at the steering column. The four loadcases were: front and rear wheels in-phase (FWIP/RWIP) and out-of-phase (FWOP/RWOP).

exhaust system. The original and optimal mount locations are shown in Fig. 10. As expected, it can be seen in this figure that the optimizer tried to position the mounts as symmetrically as possible about the TRA (triangles).

\subsection{Idle and road shake response}

The roll mode of the baseline powertrain system by itself was at $17.4 \mathrm{~Hz}$. Here the mount isolators are attached to the ground. In the full vehicle FE model, the roll frequency of the powertrain dropped down to $13.5 \mathrm{~Hz}$ due to the compliance of the frame at the mount attachments, and added inertia effect of the driveline. The front suspension hop and tramp mode of the vehicle were at 9.6 and $12.1 \mathrm{~Hz}$, respectively. In order for the powertrain roll mode to work as a tuned absorber, one needs to place it at $12 \mathrm{~Hz}$. Therefore, in ES optimization, a constraint of $15 \mathrm{~Hz}$, on the roll mode of the powertrain by itself, was defined so that a roll mode at $12 \mathrm{~Hz}$ in the full vehicle model could be obtained. In Fig. 9, it can be seen that the ES optimizer successfully placed the roll mode exactly at $12 \mathrm{~Hz}$ and decoupled the CG response in the full vehicle model. The vehicle idle shake responses at the steering column and the seat for $1^{\text {st }}, 1.5,2^{\text {nd }}, 2.5$ and $3^{r d}$ order of the engine are shown in Figs 11-12. The response values were normalized with respect to that of the baseline in both plots. The decoupled low roll solution obtained from ES, improved the idle response for all orders, particularly, 1.5 and $2^{n d}$. The objective of the optimization exercise was to improve idle quality without sacrificing road-shake performance. In Figs 13-14, the road-shake response of the vehicle at steering column and seat bolt location are shown for the standard load cases. Except for a slight degradation in the rear-wheels-out-of-phase (RWOP) loadcase, ES optimized system improved road-shake performance appreciably for all remaining loadcases. Figures 13-14 show that placement of the roll mode exactly at the front suspension tramp mode significantly reduced the response due to front-wheels-out-of-phase (FWOP) loadcase. 

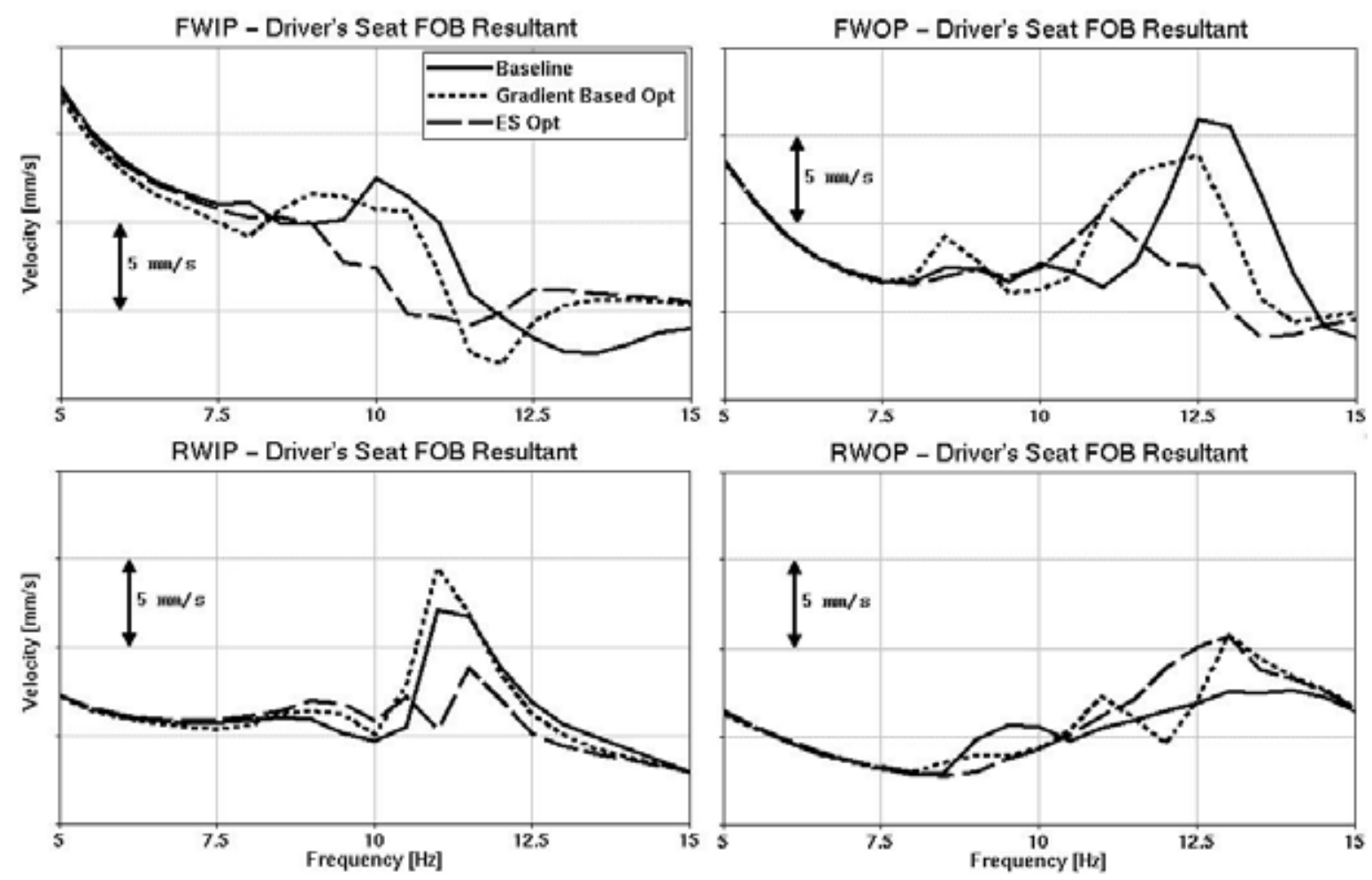

Fig. 14. Road shake response of the full-vehicle system at the driver's seat front-out-board (FOB) bolt location.

\section{Conclusions}

In this study, TRA decoupling was cast as an optimization problem so that one could obtain a decoupled roll mode of the powertrain and adjust the corresponding frequency to improve idle and road shake response of a 4 WD SUV. Both the objectives were met by implementing Evolutionary Strategies to solve a modified objective function that not only decouples the roll mode but also places it at desired frequency. The lower bounds of mounting parameters, were carefully chosen so that load bearing capacity and durability of the mounting system was not compromised. Under extreme conditions such as wide-open-throttle and sudden vehicle maneuvering, the mount isolator rates can be driven to non-linear range due to large excursions. This undesirable condition, commonly referred to as "rate build-up" can lead to grounding of the rubber mounts rendering them useless as vibration isolators. In this study, all the mount isolator stiffness values were kept within the linear range of typical force-deflection characteristics of production powertrain mounts to avoid this condition.

The Evolutionary Strategies algorithm implemented in this study, outperformed a standard gradient-based algorithm, in terms of robustness, ease of implementation, ability to locate multiple local minima and the global minimum and last but not least, offer a population of feasible solutions. Since the gradient-based optimizer used in this study was part of a commercial FE solver running on a massively parallelized supercomputer system while the ES optimization code was run on a stand-alone HP workstation in serial mode, performance of the algorithms could not be directly compared. However, in either case, the final results were obtained in less than one hour. A parallelized version of the ES code is expected to run significantly faster. As mentioned earlier, the algorithm, albeit not implemented here, can also be extended by implementing the Sequential Niche Technique, or the Species Conserving GA [25,26], to automatically locate all the relative minima. In general, for this problem, any of the standard evolutionary algorithms (GA, ES or EP), is expected to perform much better than gradient based algorithms. 


\section{Acknowledgement}

The authors would like to thank Mr. Suresh Nagamangala, (Supervisor, Truck NVH CAE, DaimlerChrysler Corp.), and Mr. Hamid Keshtkar, (Manager, Truck CAE, DaimlerChrysler Corp.) for their encouragement and support for this study.

\section{References}

[1] J.A. Cogswell and D.E. Malen, Engine mount for integral body vehicle, SAE International Congress and Exposition, Detroit, Michigan (830258), 1983.

[2] T. Jeong and R. Singh, Analytical methods of decoupling the automotive engine torque roll axis, Journal of Sound and Vibration 234(1) (2000), 85-114.

[3] K. Blackley, MSC/NASTRAN Basic Dynamic Analysis User's Guide, The MacNeal-Schwendler Corporation, Los Angeles, CA, 1995.

[4] D.B. Fogel, T. Back and Z. Michaelwicz, Evolutionary Computation 1: Basic Algorithms and Operators, (first edition), Institute of Physics Publishing, Bristol and Philadelphia, 2000.

[5] D.E. Goldberg, Genetic Algorithms in Search Optimization and Machine Learning, (first edition), Addison-Wesley Publishing Company, Massachusetts, 1989.

[6] Z. Michaelwicz, Genetic Algorithms + Data Structures = Evolution Programs, (third edition), Springer-Verlag, New York, 1999.

[7] Z. Michaelwicz and D.B. Fogel, How To Solve It: Modern Heuristics, (third edition), Springer-Verlag, Berlin, 2002.

[8] H.P. Schwefel, Evolution and Optimum Seeking, (first edition,), John Wiley, Chichester, UK, 1995.

[9] T. Back, Evolutionary Algorithms in Theory and Practice, (first edition), Oxford University Press, Oxford and New York, 1996.

[10] A. Farshidianfar and M. Ebrahimi, Optimization of vehicle driveline vibrations using genetic algorithm (ga), SAE Noise and Vibration Conference, Traverse City, Michigan, (2001-01-1502), 2001.

[11] T. Sakai, M. Iwahara, Y. Shirai and I. Hagiwara, Optimum engine mounting layout by genetic algorithm, SAE Internal Truck and Bus Meeting and Exhibition, Chicago, Illinois, (2001-01-2810), 2001.

[12] D. Hartog, Mechanical Vibrations, (first edition), Dover Publishing Inc., New York, 1985.

[13] C.E. Crede and C.M. Harris, eds, Shock and Vibration Handbook, (second edition), McGraw-Hill, New York, 1961.

[14] C.Q. Liu, A computerized optimization method of engine mounting system, SAE Noise and Vibration Conference, Traverse City, Michigan, (2003-01- 1461), 2003.

[15] C.Q. Liu, Dynamount user's manual, DaimlerChrysler Internal Report, 2002.

[16] L. Meirovitch, Analytical Methods in Vibrations, (first edition, ), Macmillan Publishing Co., Inc., New York, 1967.

[17] M. Chargin and H. Miura, A Exible Formulation for Multi-Objective Design Problems, Proceedings of The 3rd International Conference on High Performance Computing, Cray Research Inc., 1997, 147-159.

[18] J.E. Taylor and M.P. Bendsoe, An interpretation for min-max structural design problems including a method for relaxing constraints, Internal Journal of Solids and Structures 20(4) (1984), 301-314.

[19] G.N. Vanderplaats, Numerical Optimization Techniques for Engineering Design: with Applications, (first edition), McGraw-Hill, Inc., New York, 1984

[20] D.B. Fogel, T. Back and Z. Michaelwicz, eds, Evolutionary Computation 2: Advanced Algorithms and Operators, (first edition,), Institute of Physics Publishing, Bristol and Philadelphia, 2000.

[21] L. Meirovitch, Principles and techniques of Vibrations, ( first edition,), Prentice Hall, New Jersey, 1997.

[22] W.H. Press, S.A. Teukolsky, W.T. Vetterling and B.P. Flannery, Numerical Recipes in C. Cambridge University Press, (second edition,), New York, 1999.

[23] R.R. Craig and M.C.C. Bampton, Coupling of substructures for dynamic analysis, AAIA Journal 6(7) (1968), 1313-1319.

[24] T. Masters, Practical Neural Network Recipes in C++, (first edition,), Morgan Kaufmann, San Francisco, CA, 1993.

[25] D. Beasley and D.R. Bull, A sequential niche technique for multimodal function optimization, Evolutionary Computation 1(2) (1993), $101-125$.

[26] J. Li, M.E. Balazs, G.T. Parks and P.J. Clarkson. A species conserving genetic algorithm for multimodal function optimization, Evolutionary Computation 10(3) (2002), 207-234-125. 

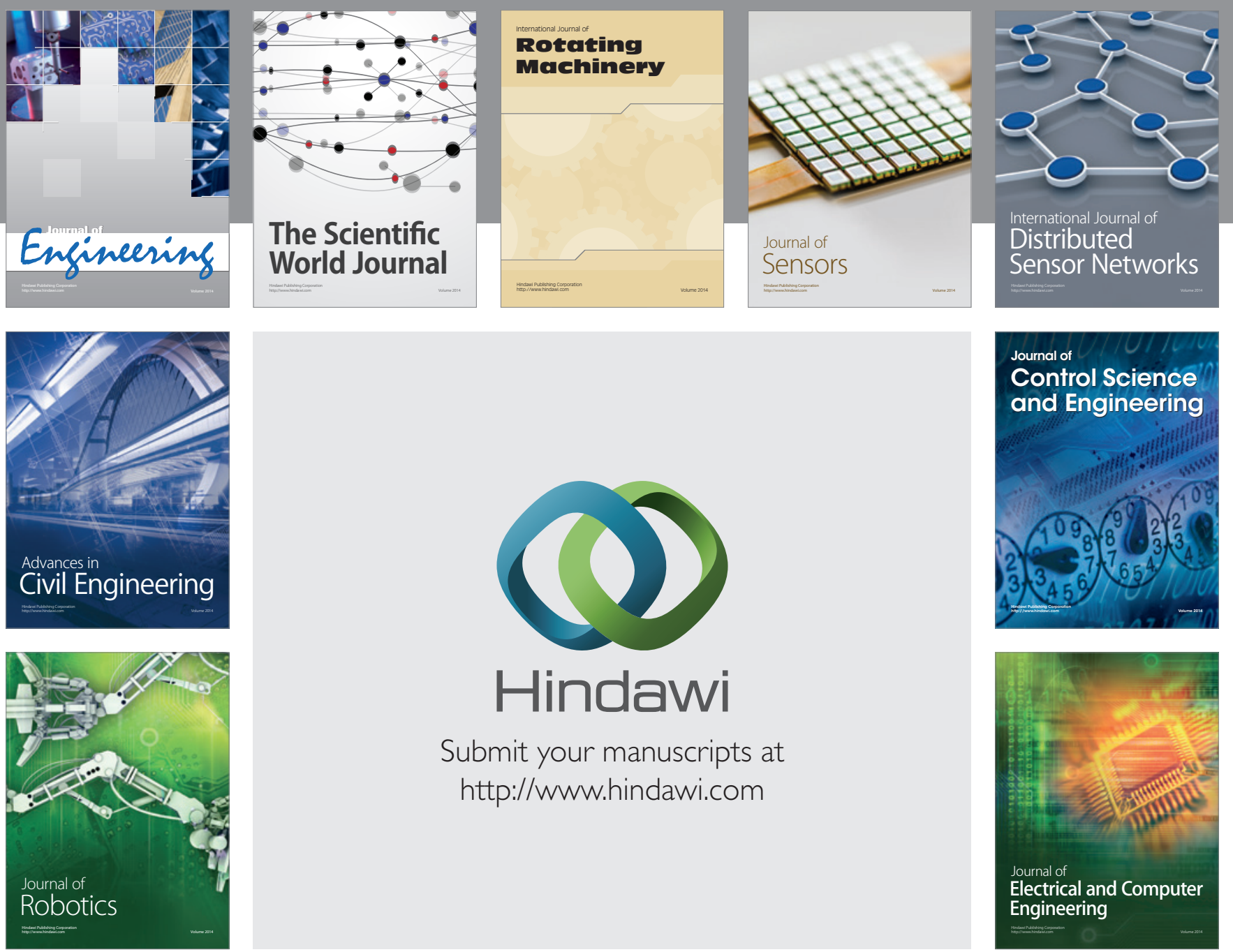

Submit your manuscripts at

http://www.hindawi.com
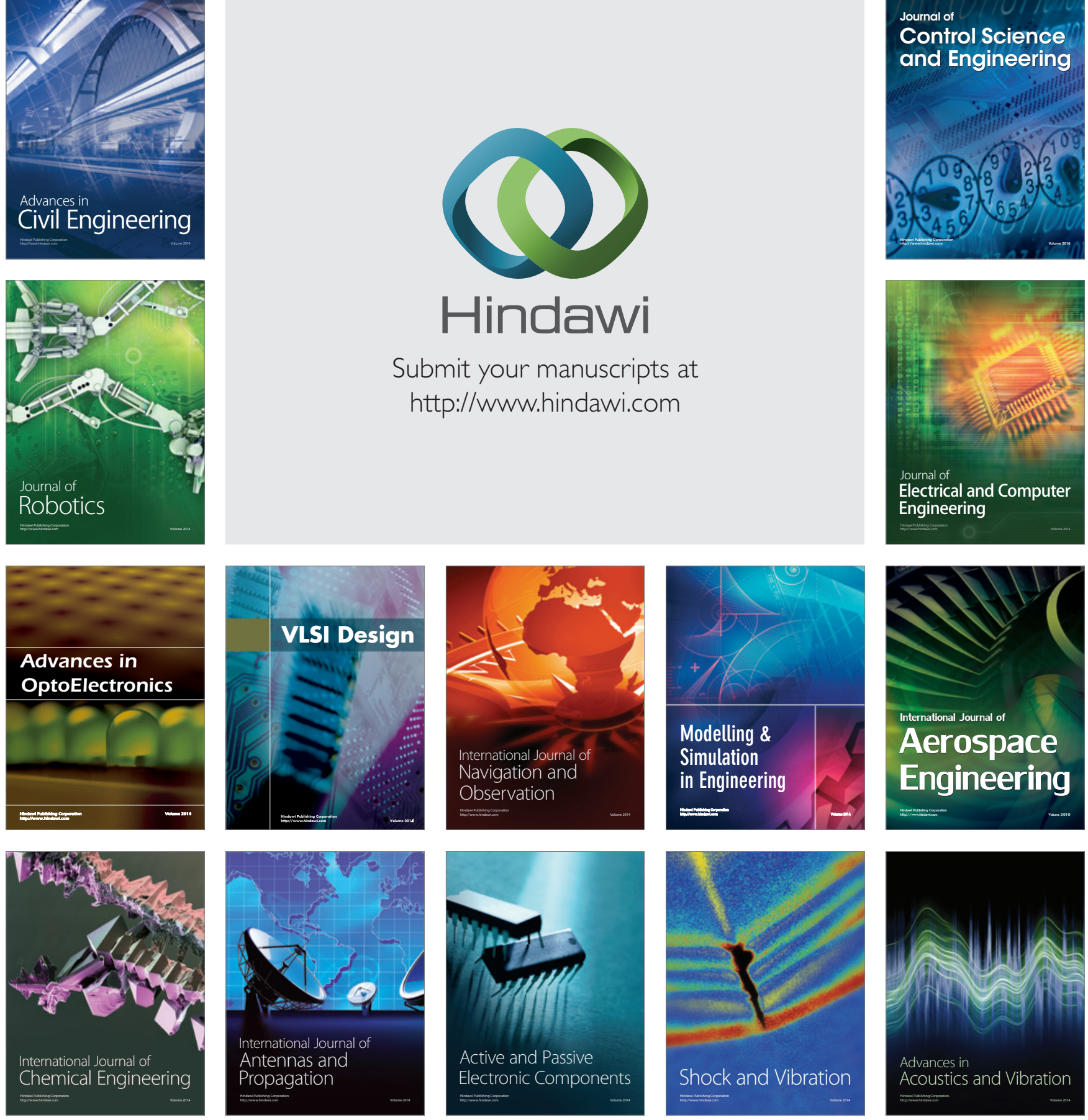